Bull. Austral. Math. Soc.

VoL. 68 (2003) [303-316]

\title{
THE GENERALISED HADAMARD INEQUALITY, $g$-CONVEXITY AND FUNCTIONAL STOLARSKY MEANS
}

\author{
E. Neuman, C.E.M. Pearce, J. PeČarić and V. Šimić
}

\begin{abstract}
We explore the role of weighted functional Stolarsky means in providing bounds in generalised Hadamard-type inequalities for $g$-convex functions. Refinements are given for Levinson's inequality and the generalised Hadamard inequality. Applications are made to multivariate weighted functional Stolarsky means.
\end{abstract}

\section{Introduction AND Preliminaries}

Weighted functional Stolarsky means have turned out to be a powerful unifying concept, subsuming a variety of classes of means appearing in the earlier literature. They have also a role as bounds in generalised Hadamard-type inequalities that apply to $g$-convex and $g$-concave functions. In this paper we explore and develop these interrelated motifs, connecting and generalising a number of results in the literature.

To introduce the concepts used in the paper, first let

$$
E_{n-1}=\left\{\left(u_{1}, \ldots, u_{n-1}\right): u_{i} \geqslant 0(1 \leqslant i \leqslant n-1), u_{1}+\cdots+u_{n-1} \leqslant 1\right\}
$$

denote the Euclidean simplex and for $u=\left(u_{1}, \ldots, u_{n-1}\right) \in E_{n-1}$, put $u_{n}:=1-\sum_{i=1}^{n-1} u_{i}$. Throughout the paper $n \geqslant 2$ and $\mu$ is an arbitrary probability measure on $E_{n-1}$. The weights $\mu_{i}(1 \leqslant i \leqslant n)$ of the measure $\mu(\cdot)$ are defined by

$$
\mu_{i}:=\int_{E_{n-1}} u_{i} d \mu(u)
$$

and so $\mu_{i} \geqslant 0$ with $\sum_{i=1}^{n} \mu_{i}=1$ and $\left(\mu_{1}, \ldots, \mu_{n}\right)$ may be taken as a set of probability weights.

We denote by $x=\left(x_{1}, \ldots, x_{n}\right)$ a real $n$-tuple with

$$
x_{\min }:=\min (x)<\max (x)=: x_{\max }
$$

Received 28th April, 2003

Copyright Clearance Centre, Inc. Serial-fee code: 0004-9727/03 \$A2.00+0.00. 
and put $\mathcal{J}=\left[x_{\min }, x_{\max }\right]$. The inner product of vectors $x, y \in \mathbb{R}^{n}$ will be denoted by $x \cdot y$. Let $f: \mathcal{J} \rightarrow \mathbb{R}$ be convex. The inequality

$$
f\left(\sum_{i=1}^{n} \mu_{i} x_{i}\right) \leqslant \int_{E_{n-1}} f(u \cdot x) d \mu(u) \leqslant \sum_{i=1}^{n} \mu_{i} f\left(x_{i}\right)
$$

has been established in [5, Theorem 4.2] and will be referred to as the generalised Hadamard inequality. Both inequalities in (1.1) are reversed if $f$ is concave on $\mathcal{J}$. When $n=2$ and $\mu$ is Lebesgue measure, (1.1) simplifies to the classical Hadamard inequality

$$
f\left(\frac{x_{1}+x_{2}}{2}\right) \leqslant \frac{1}{x_{1}-x_{2}} \int_{x_{2}}^{x_{1}} f(t) d t \leqslant \frac{1}{2}\left[f\left(x_{1}\right)+f\left(x_{2}\right)\right] .
$$

Let $f, g: \mathcal{J} \rightarrow \mathbb{R}$. Following [8] the weighted functional Stolarsky mean $m_{f, g}(x ; \mu)$ of $x$ is defined by

$$
m_{f, g}(x ; \mu)=f^{-1}\left[\int_{E_{n-1}} f \circ g^{-1}(u \cdot G) d \mu(u)\right]
$$

where $G=\left(g\left(x_{1}\right), \ldots, g\left(x_{n}\right)\right)$.

To guarantee that the operations involved are all well-defined, this definition presupposes that the functions $f$ and $g$ are continuous and strictly monotone, an assumption that will be implicit in the remainder of this section and in Sections 2 and 5 . In some situations (as here), $f$ and $g$ will be taken to act on $\mathcal{J}$, in others on the range of a further function $h$ (which we shall also assume to be continuous) defined on $\mathcal{J}$ or an interval $[a, b] \supseteq \mathcal{J}$.

Weighted functional Stolarsky means subsume a number of classes of means studied in the literature. Thus for $g(t)=t$, the mean $m_{f, g}$ simplifies to the functional mean discussed in [3] and [12]. Again, for $r \in \mathbb{R}$ define $e_{r}: \mathcal{J} \rightarrow \mathbb{R}$ by

$$
e_{r}(t):= \begin{cases}t^{r} & r \neq 0, \\ \ln t & r=0,\end{cases}
$$

which may be done provided $\mathcal{J}$ is an interval of positive numbers. The weighted Stolarsky-Tobey mean $E_{r, s}$ defined in [10] can be expressed in terms of $m_{f, g}$ by

$$
E_{r, s}(x ; \mu)=m_{e_{s-r}, e_{r}}(x ; \mu)
$$

for $r, s \in \mathbb{R}$. In particular, $L(x ; \mu):=E_{1,0}(x ; \mu)=m_{e_{-1}, e_{1}}(x ; \mu)$ is the logarithmic mean defined by Pittenger $[11], \mathcal{L}(x ; \mu):=E_{0,1}(x ; \mu)=m_{e_{1}, \ln }(x ; \mu)$ the logarithmic mean introduced by Neuman $[6]$ and $I(x ; \mu):=E_{1,1}(x ; \mu)=m_{\ln , e_{1}}(x ; \mu)$ the weighted 
identric mean considered in [13]. When $n=2$ and $\mu$ is Lebesgue measure, (1.3) becomes the unweighted (that is, uniformly weighted) Stolarsky mean $E_{r, s}(x, y)$ (see $[9,14])$.

Finally we introduce $g$-convexity, the subject of Section 2. Here it is convenient to deviate from our convention elsewhere and take $x$ to be a real number rather than an $n$-tuple. The following definition appears in [9].

Definition 1.1: Suppose $h:[a, b] \rightarrow \mathbb{R}$ and that $g$ is defined on the range of $h$. The function $h$ is said to be $g$-convex if the inequality

$$
h(\lambda x+(1-\lambda) y) \leqslant g^{-1}[\lambda(g \circ h)(x)+(1-\lambda)(g \circ h)(y)]
$$

applies for all $x, y \in[a, b]$ and $\lambda \in[0,1]$. The function $h$ is said to be $g$-concave if the reverse inequality holds.

We shall make use of the following useful property of $g$-convexity.

Proposition 1.2. Suppose $h$ is $g$-convex on $[a, b]$ and let $0 \leqslant \lambda_{i} \leqslant 1$ and $\phi_{i} \geqslant 0$ with $x_{i}, y_{i} \in[a, b](i=1, \ldots, m)$. Then

$$
\begin{aligned}
& g^{-1}\left[\sum_{i=1}^{m} \phi_{i} g \circ h\left(\lambda_{i} x_{i}+\left(1-\lambda_{i}\right) y_{i}\right)\right] \\
& \leqslant g^{-1}\left[\sum_{i=1}^{m} \phi_{i}\left[\lambda_{i} g \circ h\left(x_{i}\right)+\left(1-\lambda_{i}\right) g \circ h\left(y_{i}\right)\right]\right] .
\end{aligned}
$$

The inequality is reversed if $h$ is $g$-concave.

Proof: Suppose $h$ is $g$-convex. By the standing assumption that $g$ is strictly monotone and from Definition 1.1,

$$
g \circ h\left(\lambda_{i} x_{i}+\left(1-\lambda_{i}\right) y_{i}\right) \leqslant \lambda_{i} g \circ h\left(x_{i}\right)+\left(1-\lambda_{i}\right) g \circ h\left(y_{i}\right)
$$

if $g$ is increasing, with the reverse inequality if $g$ is decreasing. Hence

$$
\sum_{i=1}^{m} \phi_{i} g \circ h\left(\lambda_{i} x_{i}+\left(1-\lambda_{i}\right) y_{i}\right) \leqslant \sum_{i=1}^{m} \phi_{i}\left[\lambda_{i} g \circ h\left(x_{i}\right)+\left(1-\lambda_{i}\right) g \circ h\left(y_{i}\right)\right]
$$

in the case of $g$ increasing, with the reverse inequality if $g$ is decreasing. It follows that (1.5) holds in either case.

A similar argument applies when $h$ is $g$-concave.

The paper is organised as follows. Inequalities of Hadamard type for $g$-convex functions are derived in Section 2. Some results of this section provide generalisations of those established in [8]. Section 3 deals with a refinement of Levinson's inequality. The main result of this section can be regarded as an inequality of Hadamard type. Two refinements of the generalised Hadamard inequality (1.1), with the probability measure being Dirichlet measure, are derived in Section 4. In Section 5 we give two inequalities for the weighted functional Stolarsky means generated by Dirichlet measure. 


\section{INEQUALITIES FORg-CONVEX FUNCTIONS}

This section deals with inequalities of Hadamard type for $g$-convex functions, providing generalisations and improvements of results derived in [8] and [15]. We shall make use of weighted functional Stolarsky means with $n=2$. For facile interplay between these means and $g$-convexity, it is convenient to make a change of variable so that instead of $\mu$ acting on $E_{1}$ we have a probability measure $\rho$ acting on an interval $[a, b]$. We may recast the definition of a bivariate weighted Stolarsky mean as follows. Throughout this section $\theta(t):=(t-a) /(b-a)$ and

$$
\alpha:=\int_{a}^{b} \theta(t) d \rho(t)
$$

DEFINITION 2.1: Suppose $x$ and $y$ are real numbers and $f$ and $g$ are defined on the interval joining them. The weighted functional Stolarsky mean (with respect to the functions $f, g$, the interval $[a, b]$ and the probability measure $\rho$ on it) is given by

$$
m_{f, g}(x, y ; \rho)=f^{-1}\left\{\int_{a}^{b} f \circ g^{-1}[\theta(t) g(y)+(1-\theta(t)) g(x)] d \rho(t)\right\} \text {. }
$$

In the case of uniform probability measure $\rho=\theta$ on $[a, b]$, it is convenient to suppress $\rho$ and write $m_{f, g}(x, y ; \rho)$ as $m_{f, g}(x, y)$.

ThEOREM 2. Suppose that $h$ is defined on $[a, b]$ and let $f$ be defined on the range of $h$. We define $K:[0,1] \rightarrow \mathbb{R}$ by

$$
\begin{aligned}
K(t):=f^{-1}\left\{\int_{a}^{b} f \circ g^{-1}(\theta(x) g \circ h(t b+(1-t) x)\right. \\
\\
+(1-\theta(x)) g \circ h(t a+(1-t) x)) d \rho(x)\} .
\end{aligned}
$$

Then

$$
K(0)=f^{-1}\left\{\int_{a}^{b} f \circ h(x) d \rho(x)\right\}, \quad K(1)=m_{f, g}(h(a), h(b) ; \rho) .
$$

If $h$ is $g$-convex, then $K(\cdot)$ is monotone nondecreasing on $[0,1]$. If $h$ is $g$-concave, $K(\cdot)$ is monotone nonincreasing on $[0,1]$.

Proof: The values for $K(0)$ and $K(1)$ are immediate. Suppose that $h$ is $g$ convex. Let $x \in[a, b]$ and assume that $0 \leqslant s \leqslant t \leqslant 1$. By elementary algebra we have

$$
\begin{aligned}
& s b+(1-s) x=\lambda_{1} x_{1}+\left(1-\lambda_{1}\right) y_{1} \\
& s a+(1-s) x=\lambda_{2} x_{2}+\left(1-\lambda_{2}\right) y_{2}
\end{aligned}
$$


where

$$
\lambda_{1}=\frac{b t-b s+s x-t x}{t(b-a)}, \quad \lambda_{2}=\frac{b t-a s+s x-t x}{t(b-a)},
$$

$x_{1}=x_{2}=t a+(1-t) x$ and $y_{1}=y_{2}=t b+(1-t) x$.

An application of Proposition 1.2 with $m=2, \phi_{1}=\theta(x), \phi_{2}=1-\theta(x)$ gives

$$
\begin{aligned}
g^{-1}( & \left.\frac{x-a}{b-a} g \circ h(s b+(1-s) x)+\frac{b-x}{b-a} g \circ h(s a+(1-s) x)\right) \\
& \leqslant g^{-1}\left(\sum_{i=1,2} \phi_{i} \lambda_{i} g \circ h(t a+(1-t) x)+\sum_{i=1,2} \phi_{i}\left(1-\lambda_{i}\right) g \circ h(t b+(1-t) x)\right) \\
& =g^{-1}\left(\frac{x-a}{b-a} g \circ h(t b+(1-t) x)+\frac{b-x}{b-a} g \circ h(t a+(1-t) x)\right) .
\end{aligned}
$$

Since $f$ is strictly monotone, the operator $f^{-1}\left(\int_{a}^{b} f(\cdot) d \rho(x)\right)$ is monotone nondecreasing. Applying this yields $K(s) \leqslant K(t)$.

The proof with $g$-concavity is similar.

Corollary 2.3. Let $h$ be defined on $[a, b]$ and $f$ defined on the range of $h$. If $h$ is $g$-convex, then

$$
f^{-1}\left[\int_{a}^{b} f \circ h(t) d \rho(t)\right] \leqslant m_{f, g}(h(a), h(b) ; \rho)
$$

The inequality is reversed if $h$ is $g$-concave.

This result was established in [8] for the case $\rho=\theta$ as Theorem 3.2.

The next result provides bounds on bivariate weighted functional Stolarsky means.

THEOREM 2.4. Suppose that $f, g: \mathcal{J} \rightarrow \mathbb{R}$. If either

(i) $f \circ g^{-1}$ is convex and $f$ decreasing, or

(ii) $f \circ g^{-1}$ is concave and $f$ increasing,

Then

$$
f^{-1}\{\alpha f(y)+(1-\alpha) f(x)\} \leqslant m_{f, g}(x, y ; \rho) \leqslant g^{-1}\{\alpha g(y)+(1-\alpha) g(x)\} .
$$

Both inequalities are reversed if either

(iii) $f \circ g^{-1}$ is convex and $f$ increasing, or

(iv) $f \circ g^{-1}$ is concave and $f$ decreasing. 
Proof: Assume that $f \circ g^{-1}$ is convex (respectively concave). By Jensen's inequality for integrals we have that

$$
\begin{gathered}
\int_{a}^{b} f \circ g^{-1}[\theta(t) g(y)+(1-\theta(t)) g(x)] d \rho(t) \\
\stackrel{2}{\geqslant} f \circ g^{-1}\left[\int_{a}^{b}[\theta(t) g(y)+(1-\theta(t)) g(x)] d \rho(t)\right] \\
=f \circ g^{-1}[\alpha g(y)+(1-\alpha) g(x)]
\end{gathered}
$$

which gives the second inequality in (2.1) and its reverse. For the first inequality, assume $f \circ g^{-1}$ is convex (respectively concave). By Jensen's discrete inequality,

$$
\begin{aligned}
& \int_{a}^{b} f \circ g^{-1}[\theta(t) g(y)+(1-\theta(t)) g(x)] d \rho(t) \stackrel{(\geqslant)}{\leqslant} \int_{a}^{b}[\theta(t) f(y)+(1-\theta(t)) f(x)] d \rho(t) \\
& =\alpha f(y)+(1-\alpha) f(x),
\end{aligned}
$$

which gives the desired result.

Corollary 2.3 and Theorem 2.4 can be used to obtain the following generalisation of [8, Theorem 3.3].

THEOREM 2.5. Let $h:[a, b] \rightarrow \mathbb{R}$ be continuous and $f$ defined on the range of $h$. If $h$ is $g$-convex and either (i) or (ii) of Theorem 2.4 applies, then

$$
\begin{aligned}
f^{-1}\left\{\int_{a}^{b} f \circ h(t) d \rho(t)\right\} & \leqslant m_{f, g}(h(a), h(b) ; \rho) \\
& \leqslant g^{-1}\{\alpha g \circ h(b)+(1-\alpha) g \circ h(a)\} .
\end{aligned}
$$

Both inequalities are reversed if $h$ is $g$-concave and either (iii) or (iv) of Theorem 2.4 applies.

Moreover, if $h$ is $g$-convex and either (iii) or (iv) of Theorem 2.4 applies, then

$$
\begin{aligned}
f^{-1}\left[\int_{a}^{b} f \circ h(t) d \rho(t)\right] & \leqslant m_{f, g}(h(a), h(b) ; \rho) \\
& \leqslant f^{-1}[\alpha f \circ h(b)+(1-\alpha) f \circ h(a)]
\end{aligned}
$$

Both inequalities are reversed if $h$ is $g$-concave and either (i) or (ii) of Theorem 2.4 applies.

Proof: The first inequality in each displayed relation comes from Corollary 2.3 and the second from Theorem 2.4. 
We now link our ideas with the notion of $r$-convexity and $r$-concavity. If $h$ is positive and $r$-convex on $[a, b]$, then

$$
h(\lambda x+(1-\lambda) y) \leqslant m_{r}(h(x), h(y) ; \lambda)
$$

for $\lambda \in[0,1]$ and all $x, y \in[a, b]$, where the weighted power mean $m_{r}$ of order $r \in \mathbb{R}$ of positive numbers $v, w$ (with nonnegative weights $\lambda, 1-\lambda$ ) is given by

$$
m_{r}(v, w ; u):= \begin{cases}\left(\lambda v^{r}+(1-\lambda) w^{r}\right)^{1 / r} & \text { if } r \neq 0 \\ v^{\lambda} w^{1-\lambda} & \text { if } r=0\end{cases}
$$

We can readily show that the right-hand side of (2.3) is the same as that of (1.4) when $g=e_{r}$, so that $h$ is $g$-convex for $g=e_{r}$. Similarly a positive $r$-concave function, for which the inequality (2.3) is reversed, is $g$-concave for $g=e_{r}$.

Now choose $\rho=\theta$ and $f=e_{p}$ for $p \in \mathbb{R}$. With elementary algebra we derive

$$
K(t)=M_{p}\left(m_{r}(h \circ A(b, \cdot ; t), h \circ A(a, \cdot ; t) ; \theta(\cdot))\right)
$$

where

$$
A(x, y ; \lambda):=\lambda x+(1-\lambda) y
$$

denotes the weighted arithmetic mean of positive numbers $x, y$ with weights $\lambda$ and $1-\lambda(\lambda \in[0,1])$, and

$$
M_{p}(f):=\left\{\begin{array}{ll}
{\left[\frac{1}{b-a} \int_{a}^{b}(f(t))^{p} d t\right]^{1 / p}} & \text { if } p \neq 0 \\
\exp \left(\frac{1}{b-a} \int_{a}^{b} \ln f(t) d t\right) & \text { if } p=0
\end{array},\right.
$$

that is, $M_{p}(f)=e_{p}^{-1} \int_{a}^{b} e_{p} \circ f(t) d \theta(t)$, is the integral power mean of order $p \in \mathbb{R}$ of a positive function $f$ on $[a, b]$. This gives the following result.

Corollary 2.6. Suppose that $h$ is positive on $[a, b](x \in[a, b])$ and let $K$ : $[0,1] \rightarrow \mathbb{R}$ be defined by (2.4). Then

$$
K(0)=M_{p}(h) \text { and } K(1)=E_{r+p, r}(h(a), h(b))
$$

If $h$ is $r$-convex, then $K$ is monotone increasing on $[0,1]$. If $h$ is $r$-concave, $K$ is monotone decreasing on $[0,1]$.

This result was established recently in [15]. 


\section{AN IMPROVEMENT OF LEVINSON'S INEQUALITY}

Levinson [4] has given the following important generalisation of the Ky Fan inequality.

THEOREM 3.1. Suppose $f$ has a third-order derivative on $] 0,2 a\left[\right.$ and $f^{\prime \prime \prime}(t)$ $\geqslant 0$. Let $u_{i}>0$ for all $i \in\{1, \ldots, n\}$. Then for $\left.x_{i} \in\right] 0, a[$, we have

$$
\sum_{i=1}^{n} u_{i} f\left(x_{i}\right)-f\left(\sum_{i=1}^{n} u_{i} x_{i}\right) \leqslant \sum_{i=1}^{n} u_{i} f\left(2 a-x_{i}\right)-f\left(\sum_{i=1}^{n} u_{i}\left(2 a-x_{i}\right)\right) .
$$

If $f^{\prime \prime \prime}(t)>0$, the inequalities become equalities if and only if $x_{1}=\cdots=x_{n}$.

This result may be usefully viewed in terms of 3-convexity. We remark that the notion of $n$-convexity here is different from that of the previous section.

DEFINITION 3.2. A function $f:[a, b] \rightarrow \mathbb{R}$ is said to be $n$-convex $(n \geqslant 0)$ on $[a, b]$ if and only if, for all choices of $n+1$ distinct points in $[a, b]$, the nth-order divided difference $\left[x_{0}, \ldots, x_{n}\right] f$ of $f$ is nonnegative.

Bullen [1] has shown that (3.1) applies if $f$ is a 3-convex function. Since (3.1) may be rearranged as

$$
f\left(\sum_{i=1}^{n} u_{i}\left(2 a-x_{i}\right)\right)-f\left(\sum_{i=1}^{n} u_{i} x_{i}\right) \leqslant \sum_{i=1}^{n} u_{i}\left[f\left(2 a-x_{i}\right)-f\left(x_{i}\right)\right],
$$

we thus have the following.

Lemma 3.3. If $f:[0,2 a] \rightarrow \mathbb{R}$ is 3-convex, then $f(2 a-t)-f(t)$ is convex on ]0,a[.

By combining this result with (1.1), we derive a generalisation of the Levinson inequality which has generalised-Hadamard form.

THEOREM 3.4. Let $f:[0,2 a] \rightarrow \mathbb{R}$ be 3-convex. Then for $\left.x_{i} \in\right] 0, a[$, we have

$$
\begin{aligned}
f\left(\sum_{i=1}^{n} \mu_{i}\left(2 a-x_{i}\right)\right)-f\left(\sum_{i=1}^{n} \mu_{i} x_{i}\right) & \leqslant \int_{E_{n-1}}[f(u \cdot(2 a-x))-f(u \cdot x)] d \mu(u) \\
& \leqslant \sum_{i=1}^{n} \mu_{i}\left[f\left(2 a-x_{i}\right)-f\left(x_{i}\right)\right] .
\end{aligned}
$$

EXAmple. Suppose $f(t)=\ln t$, so that $f(t)$ is 3-convex on $] 0,1]$. Let $\left.\left.x_{i} \in\right] 0,1 / 2\right]$ $(i=1, \ldots, n)$, where $n \geqslant 2$. From (3.2) with $a=1 / 2$ we obtain

$$
\frac{\prod_{i=1}^{n} x_{i}^{\mu_{i}}}{\prod_{i=1}^{n}\left(1-x_{i}\right)^{\mu_{i}}} \leqslant \frac{I\left(x_{1}, \ldots, x_{n} ; \mu\right)}{I\left(1-x_{1}, \ldots, 1-x_{n} ; \mu\right)} \leqslant \frac{\sum_{i=1}^{n} \mu_{i} x_{i}}{\sum_{i=1}^{n} \mu_{i}\left(1-x_{i}\right)}
$$


where $I\left(x_{1}, \ldots, x_{n} ; \mu\right)=I(x ; \mu)=E_{1,1}(x ; \mu)=m_{\mathfrak{l n}, e_{1}}(x ; \mu)$ is the weighted identric mean.

This inequality has been established in [13]. With the choice $d \mu(u)=(n-1) ! d u_{1} \cdots d u_{n-1}$, it is a refinement of the Ky Fan inequality

$$
\frac{G_{n}}{G_{n}^{\prime}} \leqslant \frac{I_{n}}{I_{n}^{\prime}} \leqslant \frac{A_{n}}{A_{n}^{\prime}}
$$

for unweighted means. Here $A_{n}, G_{n}$ and $I_{n}$ represent respectively the arithmetic, geometric and identric means of $x_{1}, \ldots, x_{n}$ and $A_{n}^{\prime}, G_{n}^{\prime}$ and $I_{n}^{\prime}$ those for $1-x_{1}, \ldots, 1$ $-x_{n}$.

\section{Dirichlet AVERAges}

In this section we make use of the particular choice $\mu=\mu_{b}$, Dirichlet measure. For $b=\left(b_{1}, \ldots, b_{n}\right) \in \mathbb{R}_{+}^{n}$, this is given by

$$
d \mu_{b}(u)=\frac{1}{B(b)} \prod_{j=1}^{n} u_{j}^{b_{j}-1} d u
$$

where $d u=d u_{1} \ldots d u_{n-1}$ and $B(b)$ is the multivariate beta function. The associated weights are

$$
\mu_{i}=b_{i} / c
$$

$(1 \leqslant i \leqslant n)$, where $c=b_{1}+\cdots+b_{n}$ (see [2, (4.4-8)]). The Dirichlet average of a function $f: \mathcal{J} \rightarrow \mathbb{R}$ is defined as

$$
F(b ; x)=\int_{E_{n-1}} f(u \cdot x) d \mu_{b}(u) .
$$

Dirichlet averages are useful in the theory of special functions (see [2]). We list below some basic properties.

(i) $F(b ; x)$ is symmetric in the indices $1, \ldots, n$, which label the $b$-parameters and the variables $x$.

(ii) Two or more identical variables can be replaced by a single variable if the corresponding $b$-parameters are replaced by their sum.

(iii) A vanishing $b$-parameter can be omitted along with the corresponding variable.

(iv) The Dirichlet average $F(b ; x)$ is holomorphic in the elements of $b$ and $x$ on its domain of definition. 
When $\mu=\mu_{b},(1.1)$ reduces to

$$
f\left(\sum_{i=1}^{n} \mu_{i} x_{i}\right) \leqslant F(b ; x) \leqslant \sum_{i=1}^{n} \mu_{i} f\left(x_{i}\right),
$$

with $\mu_{i}$ as in (4.1) (see [2, Exercise 5.2-1]). Two refinements are given in this section. Applications of these results to weighted functional Stolarsky means are discussed in Section 5.

For $b \in \mathbb{R}_{+}^{n}$ and $\gamma \in \mathbb{R}$ let $b_{\gamma}=\left(b_{1}, \ldots, b_{n-1}, b_{n}+\gamma\right)$. Thus $b_{0}=b$. Also, let $\alpha=c /(c+\gamma)$ and $\beta=1-\alpha$. If $\gamma>0$, then $\alpha>0, \beta>0$ and $\alpha+\beta=1$. We define $d=(c, \gamma)$, where $c=b_{1}+\cdots+b_{n}$ and

$$
y=\left(\alpha x_{1}+\beta x_{n}, \alpha x_{2}+\beta x_{n}, \ldots, \alpha x_{n}+\beta x_{n}\right) .
$$

ThEOREM 4.1. Let $f: \mathcal{J} \rightarrow \mathbb{R}$ be convex and suppose $\gamma>0$. Then

$$
F(b ; y) \leqslant F\left(b_{\gamma} ; x\right) \leqslant \alpha F(b ; x)+\beta f\left(x_{n}\right)
$$

and

$$
F\left(d ; \sum_{i=1}^{n} \mu_{i} x_{i}, x_{n}\right) \leqslant F\left(b_{\gamma} ; x\right) \leqslant \sum_{i=1}^{n} \mu_{i} F\left(d ; x_{i}, x_{n}\right)
$$

where $\mu_{i}$ is given by (4.1). Inequalities (4.4) and (4.5) are reversed if $f$ is concave on $\mathcal{J}$.

Proof: Let $w=\left(w_{1}, \ldots, w_{n}\right)$, where $w_{i}=t x_{i}+(1-t) x_{n}$ for $1 \leqslant i \leqslant n$ and $0 \leqslant t \leqslant 1$. Clearly $w_{n}=x_{n}$. We shall employ the result

$$
F\left(b_{\gamma} ; x\right)=\int_{0}^{1} F(b ; w) d \mu_{d}(t)
$$

(see [7, Corollary 3.4]). Here $\mu_{d}$ is Dirichlet measure on $[0,1]$ with parameters $d$ $=(c, \gamma)$. Assume that $f$ is convex on $\mathcal{J}$. For the proof of the first inequality in (4.4), we combine (4.6) with (4.2) and apply Jensen's inequality for integrals to obtain

$$
\begin{aligned}
F\left(b_{\gamma} ; x\right) & =\int_{E_{n-1}}\left[\int_{0}^{1} f(u \cdot w) d \mu_{d}(t)\right] d \mu_{b}(u) \\
& \geqslant \int_{E_{n-1}} f\left[\int_{0}^{1}(u \cdot w) d \mu_{d}(t)\right] d \mu_{b}(u) \\
& =\int_{E_{n-1}} f\left[\sum_{i=1}^{n} u_{i} \int_{0}^{1}\left(t x_{i}+(1-t) x_{n}\right) d \mu_{d}(t)\right] d \mu_{b}(u)
\end{aligned}
$$


Since

$$
\int_{0}^{1}\left(t x_{i}+(1-t) x_{n}\right) a^{j} \mu_{d}(t)=\alpha x_{i}+\beta x_{n}
$$

(see $[2,(4.4-8)])$,

$$
F\left(b_{\gamma} ; x\right) \geqslant \int_{E_{n-1}} f(u \cdot y) d \mu_{b}(u)=F(b ; y) .
$$

For the second inequality in (4.4), we utilise the convexity of $F(b ; w)$ in its variables to obtain

$$
\begin{aligned}
F(b ; w) & =F\left(b ; t x_{1}+(1-t) x_{n}, \ldots, t x_{n}+(1-t) x_{n}\right) \\
& \leqslant t F(b ; x)+(1-t) F(b ; \underbrace{x_{n}, \ldots, x_{n}}_{n \text {-times }}) .
\end{aligned}
$$

Since $F\left(b ; x_{n}, \ldots, x_{n}\right)=f\left(x_{n}\right)$ (see Property (ii)),

$$
F(b ; w) \leqslant t F(b ; x)+(1-t) f\left(x_{n}\right) .
$$

Integrating both sides of the last inequality against the Dirichlet measure $\mu_{d}$ and using (4.6), we arrive at

$$
\begin{aligned}
F\left(b_{\gamma} ; x\right) & =\int_{0}^{1} F(b ; w) d \mu_{d}(t) \\
& \leqslant F(b ; x) \int_{0}^{1} t d \mu_{d}(t)+f\left(x_{n}\right) \int_{0}^{1}(1-t) d \mu_{d}(t) \\
& =\alpha F(b ; x)+\beta f\left(x_{n}\right) .
\end{aligned}
$$

For the proof of (4.5) we utilise (4.3) with $x$ replaced by $w$ to obtain

$$
f\left[\sum_{i=1}^{n} \mu_{i}\left(t x_{i}+(1-t) x_{n}\right)\right] \leqslant F(b ; w) \leqslant \sum_{i=1}^{n} \mu_{i} f\left(t x_{i}+(1-t) x_{n}\right)
$$

Integrating each term against the Dirichlet measure $\mu_{d}$ and using (4.6) provides (4.5).

The first and last terms in (4.4) and (4.5) can be bounded below and above, respectively. We have the following.

Corollary 4.2. Define $v_{i}:=b_{i} /(c+\gamma)$ for $1 \leqslant i \leqslant n-1$ and $v_{n}$ 
$:=\left(b_{n}+\gamma\right) /(c+\gamma)$. Then under the assumptions of Theorem 4.1, we have

$$
\begin{aligned}
f\left(\sum_{i=1}^{n} v_{i} x_{i}\right) & \leqslant F(b ; y) \\
\alpha F(b ; x)+\beta f\left(x_{n}\right) & \leqslant \sum_{i=1}^{n} v_{i} f\left(x_{i}\right) \\
f\left(\sum_{i=1}^{n} v_{i} x_{i}\right) & \leqslant F\left(d ; \sum_{i=1}^{n} \mu_{i} x_{i}, x_{n}\right) \\
\sum_{i=1}^{n} \mu_{i} F\left(d ; x_{i}, x_{n}\right) & \leqslant \sum_{i=1}^{n} v_{i} f\left(x_{i}\right) .
\end{aligned}
$$

REMARK. Combining the first two inequalities in (4.7) with (4.4), we see that the latter provides a refinement of (4.3) with $b$ replaced by $b_{\gamma}$. A similar statement applies to (4.5).

Proof of Corollary 4.2: From the first inequality in (4.3),

$$
\begin{aligned}
F(b ; y) \geqslant f\left(\sum_{i=1}^{n} \mu_{i} y_{i}\right) & =f\left(\sum_{i=1}^{n} \frac{b_{i}}{c}\left(\frac{c}{c+\gamma} x_{i}+\frac{\gamma}{c+\gamma} x_{n}\right)\right) \\
& =f\left(\sum_{i=1}^{n} v_{i} x_{i}\right),
\end{aligned}
$$

giving the first inequality in (4.7). The others follow similarly.

For further refinements of (4.3) see [6, Theorem 3].

\section{INEQUALITIES FOR FUNCTIONAL STOLARSKY MEANS}

Several inequalities for weighted functional Stolarsky means have been obtained in $[8,9,10]$. In this section we utilise the results of Section 4 to derive two inequalities involving these means. We deal with the Stolarsky means defined in (1.2) when $\mu=\mu_{b}$ and we write $m_{f, g}(x ; b)$ instead of $m_{f, g}\left(x ; \mu_{b}\right)$. It follows from (1.2) and (4.2) that

$$
m_{f, g}(x ; b)=f^{-1}[H(b ; G)],
$$

where, as in the remainder of this section, $h=f^{-1} \circ g$ and $H$ denotes its Dirichlet average.

THEOREM 5.1. Suppose $h=f \circ g^{-1}$ is increasing. If either $h$ and $g$ are convex and $f$ increasing, or $h$ and $g$ concave and $f$ decreasing, then

$$
m_{f, g}(y ; b) \leqslant m_{f, g}\left(x ; b_{\gamma}\right) \leqslant f^{-1}\left[\alpha H(b ; G)+\beta f\left(x_{n}\right)\right]
$$


and

$$
m_{f, g}\left(\sum_{i=1}^{n} \mu_{i} x_{i}, x_{n} ; d\right) \leqslant m_{f, g}\left(x ; b_{\gamma}\right) \leqslant f^{-1}\left[\sum_{i=1}^{n} \mu_{i} H\left(d ; g\left(x_{i}\right), g\left(x_{n}\right)\right)\right]
$$

Inequalities (5.2) and (5.3) are reversed if either $h$ and $g$ are convex and $f$ decreasing or $h$ and $g$ concave and $f$ increasing.

REMARK. If $f^{-1}$ is convex, then the third members in (5.2) and (5.3) can be bounded above respectively by

$$
\alpha m_{f, g}(x ; b)+\beta x_{n} \quad \text { and } \quad \sum_{i=1}^{n} \mu_{i} m_{f, g}\left(x_{i}, x_{n} ; d\right) .
$$

Proof of TheOREM 5.1: Suppose first that $h$ and $g$ are convex and $f$ increasing. Making use of (4.4), with $f$ replaced by $h$ and $x$ by $G=\left(g\left(x_{1}\right), \ldots, g\left(x_{n}\right)\right)$, we obtain

$$
H(b ; Y) \leqslant H\left(b_{\gamma} ; G\right) \leqslant \alpha H(b ; G)+\beta f\left(x_{n}\right),
$$

where

$$
H(b ; Y)=\int_{E_{n-1}} h(u \cdot Y) d \mu_{b}(u)
$$

with $Y=\left(\alpha g\left(x_{1}\right)+\beta g\left(x_{n}\right), \alpha g\left(x_{2}\right)+\beta g\left(x_{n}\right), \ldots, \alpha g\left(x_{n}\right)+\beta g\left(x_{n}\right)\right)$. Since $h$ is convex and increasing, we have $H(b ; Y) \geqslant H(b ; y)$. In conjunction with (5.4), this gives

$$
H(b ; y) \leqslant H\left(b_{\gamma} ; G\right) \leqslant \alpha H(b ; G)+\beta f\left(x_{n}\right) .
$$

Since $f$ is increasing, the last inequality combined with (5.1) gives the desired result (5.2) for the case when $h$ and $g$ are convex and $f$ increasing. The remaining cases of (5.2) and (5.3) can be established similarly.

\section{REFERENCES}

[1] P.S. Bullen, 'An inequality of N. Levinson', Univ. Beograd. Publ. Elektrotehn. Fak. Ser. Mat. Fiz. 412-460 (1973), 109-112.

[2] B.C. Carlson, Special Functions of Applied Mathematics (Academic Press, New York, 1977).

[3] S.-Y. Chung, 'Functional means and harmonic functional means', Bull. Austral. Math. Soc. 57 (1998), 207-220.

[4] N. Levinson, 'Generalization of an inequality of Ky Fan', J. Math. Anal. Appl. 8 (1969), 133-134. 
[5] E. Neuman, 'Inequalities involving multivariate convex functions II', Proc. Amer. Math. Soc. 109 (1990), 965-974.

[6] E. Neuman, 'The weighted logarithmic mean', J. Math. Anal. Appl. 188 (1994), 885-900.

[7] E. Neuman, 'Dirichlet averages and their applications to Gegenbauer functions', Int. J. Math. Stat. Sci. 5 (1996), 26-42.

[8] C.E.M. Pearce, J. Pečarić and V. Šimić, 'Functional Stolarsky means', Math. Inequal. Appl. 2 (1999), 479-489.

[9] C.E.M. Pearce, J. Pečarić and V. Šimić, 'Stolarsky means and Hadamard's inequality', $J$. Math. Anal. Appl. 220 (1998), 99-109.

[10] J. Pečarić and V. Šimić, 'Stolarsky-Tobey mean in $n$ variables', Math. Inequal. Appl. 2 (1999), 325-341.

[11] A.O. Pittenger, 'The logarithmic mean in n variables', Amer. Math. Monthly 92 (1987), 282-291.

[12] F. Saidi and R. Younis, 'Hadamard and Fejér-type inequalities', Arch. Math. (Basel) 74 (2000), 30-39.

[13] J. Sandor and T. Trif, 'A new refinement of the Ky Fan inequality', Math. Inequal. Appl. 2 (1999), 529-533.

[14] K.B. Stolarsky, 'Generalizations of the logarithmic mean', Math. Mag. 48 (1975), 87-92.

[15] G-S. Yang and D-Y. Hwang, 'Refinements of Hadamard's inequality for $r$-convex functions', Indian J. Pure Appl. Math. 32 (2001), 1571-1579.

Department of Mathematics

Southern Illinois University at Carbondale

Carbondale, IL 62901-4408

United States of America

e-mail: edneuman@siu.edu

Faculty of Textile Technology

University of Zagreb

Pierottijeva 6

11000 Zagreb

Croatia

e-mail: pecaric@hazu.hr
School of Applied Mathematics

Adelaide University

Adelaide SA 5005

Australia

e-mail: cpearce@maths.adelaide.edu.au

Faculty of Textile Technology

University of Zagreb

Pierottijeva 6

11000 Zagreb

Croatia

e-mail: vidasim@ztk.ttf.hr 